\title{
The Role of TRAF4 and B3GAT1 Gene Expression in the Food Hypersensitivity and Insect Venom Allergy in Mastocytosis
}

\author{
Aleksandra Górska ${ }^{1} \cdot$ Marta Gruchała-Niedoszytko $^{2} \cdot$ Marek Niedoszytko $^{1}$. \\ Agnieszka Maciejewska ${ }^{3}$. Marta Chełmińska ${ }^{1}$ Marcin Skrzypski ${ }^{4}$. \\ Bartosz Wasąg ${ }^{5} \cdot$ Małgorzata Kaczkan $^{2} \cdot$ Magdalena Lange $^{6} \cdot$ Bogusław Nedoszytko $^{6}$. \\ Ryszard Pawłowski ${ }^{3}$ Sylwia Małgorzewicz ${ }^{2} \cdot$ Ewa Jassem $^{1}$
}

Received: 1 July 2015/Accepted: 27 January 2016/Published online: 16 April 2016

(c) The Author(s) 2016. This article is published with open access at Springerlink.com

\begin{abstract}
Mastocytosis is an uncommon disease classified as a myeloproliferative neoplasm, however, its symptoms are broad and place patients at crossroads between dermatology, hematology and allergology. Patients with mastocytosis often suffer from symptoms resulting from the activation and release of mediators from the mast cells, such as generalized itching, redness, headache, abdominal cramps, diarrhea, bone pain or arthritis, hypotension and shock. The possible severe, fatal or near fatal reactions caused by food hypersensitivity are reasons for the research focused on marker identification. The aim of the study was to analyse the gene expression differences in mastocytosis patients with and without food and drug
\end{abstract}

A. Górska and M. Gruchała-Niedoszytko share equal contribution.

The gene expression analysis was performed in the Department of Forensic Medicine and Department of Genetics of the Medical University of Gdansk.

Marta Gruchała-Niedoszytko

mg@gumed.edu.pl

1 Department of Allergology, Medical University of Gdansk, Gdańsk, Poland

2 Department of Clinical Nutrition, Medical University of Gdansk, Marii Skłodowskiej-Curie 3a, 80-210 Gdańsk, Poland

3 Department of Forensic Medicine, Medical University of Gdansk, Gdańsk, Poland

4 Department of Oncology, Medical University of Gdansk, Gdańsk, Poland

5 Department of Genetics, Medical University of Gdansk, Gdańsk, Poland

6 Department of Dermatology, Medical University of Gdansk, Gdańsk, Poland hypersensitivity and insect venom allergy (IVA). A total of 57 Caucasian patients with mastocytosis were studied [median age 41.8; range 18-77 years; 15 (26.3\%) males and $42(73.7 \%)$ females]. Quantitative RT-PCRs of 11 genes plus ribosomal 18S RNA were run. Symptoms of food hypersensitivity were found in 12 patients (21\%), including 3 patients $(13 \%)$ with cutaneous mastocytosis (CM), and $9(28 \%)$ with indolent systemic mastocytosis (ISM). IVA was confirmed in 13 patients $(22.8 \%)$ including 6 patients $(10.5 \%)$ with $\mathrm{CM}$, and 7 patients $(12.3 \%)$ with ISM. Drug hypersensitivity was diagnosed in 10 patients $(17.5 \%)$. Significant differences in the gene expression were found for TRAF4 $(p=0.008)$ in the comparison of the mastocytosis patients with and without concomitant food hypersensitivity. Furthermore significant differences were found in gene expression for B3GAT1 $(p=0.003)$ in patients with IVA compared to patients without insect sting anaphylaxis in the medical history. The expression of studied genes did not differ according to the presence of drug hypersensitivity. The TRAF4 expression was higher in mastocytosis patients with food hypersensitivity in their medical history, the B3GAT1 expression was lower in mastocytosis patients with IVA in history.

Keywords Mastocytosis - Food allergy ·

Food hypersensitivity - Drug hypersensitivity ·

Insect sting anaphylaxis

\section{Introduction}

Mastocytosis is an uncommon disease classified as a myeloproliferative neoplasm, however, its symptoms are broad and place patients at crossroads between dermatology, hematology and allergology (Valent et al. 2007, 2012). 
Dutch data suggest the increasing prevalence of the disease which may be related to greater awareness of the physicians, and tryptase measurements in patients with anaphylaxis, however, the influence of environmental factors may not be excluded (van Doormaal et al. 2013). The severity of symptoms related to the mast cell bone marrow infiltration is mild in the majority of subjects classified as cutaneous or indolent. The anaphylactic reactions prevalent in approximately half of the patients constitute principal causes of the quality of life impairment and systemic life threatening reactions (Brockow et al. 2008; Jennings et al. 2014). The most important eliciting agents in mastocytosis are insect venom, food and drug allergens, and physical factors (Brockow et al. 2008; Cifuentes et al. 2013; Górska et al. 2015). Insect sting is considered a major cause of mast cell activation in patients with mastocytosis. It is estimated that $30 \%$ of patients with mastocytosis have insect sting anaphylactic reactions (Brockow et al. 2008), which are more frequent and more severe than in general population with insect venom allergies (IVA; 1-3\%) (Ludolph-Hauser et al. 2001). Furthermore, the more severe anaphylaxis may be the effect of the activation of a cascade of intracellular tyrosine kinases: Kit, Lyn, Syk and Fyn in abnormal mast cells. However, the presence of KIT gene mutations, notably $\mathrm{D} 816 \mathrm{~V}$, detectable in more than $90 \%$ of patients with systemic mastocytosis resulting in an increased activation of mast cells, does not correlate with the severity or the prevalence of anaphylaxis (Peavy and Metcalfe 2008). The food hypersensitivity is responsible for a variety of reactions ranging from common abdominal symptoms to anaphylactic reactions. The definition of the European Academy of Allergy and Clinical Immunology describes food hypersensitivity as an abnormally strong response to a food stimulus, whereas a subgroup of immunologically mediated reactions is referred to as a food allergy (Ring 2014). Epidemiological data indicate that symptoms of food hypersensitivity are present in $17 \%$ of the general population. A thorough diagnosis using a double blind placebo control food challenge confirmed a food allergy diagnosis in $0.9 \%$ of the general population (approx. $5 \%$ of patients declaring food hypersensitivity) (Nwaru et al. 2014). The Epidemiology of Allergic Diseases study (in Poland) found the symptoms of food hypersensitivity in $13 \%$ of children aged 6-7 years, $11 \%$ of teenagers 13-14 years and 5\% in adults (Samolinski 2015). The data of the Polish Centre of the European Competence Network on Mastocytosis indicate that symptoms related to food hypersensitivity were found in $29 \%$ of mastocytosis patients (Górska et al. 2015). A significant infiltration of the mast cells may be found in various chronic inflammatory diseases (Henderson et al. 2012). The higher number of mast cells found in mastocytosis patients is also a risk factor to the symptoms caused by food rich in histamine, biogenic amines and histaminereleasing type of food (Vlieg-Boerstra et al. 2005). The possible severe, fatal or near fatal reactions caused by food hypersensitivity are reasons for the research focused on the identification of the markers which could be used to assess the risk and initiate the treatment to lower the severity of a possible reaction. Currently, several clinical markers can be used as the tryptase level, severity of skin involvement, and the prevalence of indolent systemic mastocytosis (ISM) (Brockow et al. 2008; Górska et al. 2015). However, the diagnosis based on the gene expression, common method used in oncology and hematology, may improve the diagnosis and tailor the therapy. Currently there are efforts to find a less invasive diagnostic procedure. Niedoszytko et al. (2011) found significant differences in gene expression profile in ISM patients with insect venom anaphylaxis compared to ISM patients without anaphylaxis in history. Authors insist that further studies in larger group of patients are required to validate their results for the development of a predictive tool to be used in clinical practice.

The aim of the study was to analyse the gene expression differences in mastocytosis patients with and without food and drug hypersensitivity, and IVA.

\section{Materials and Methods}

\section{Patients}

A total of 57 Caucasian patients with mastocytosis, treated at the Department of Allergology, Medical University of Gdańsk were studied [median age 41.8; range 18-77 years; $15(26.3 \%)$ males and $42(73.7 \%)$ females]. All patients underwent standard diagnostic procedures in accordance with the WHO and ECNM (European Competence Network on Mastocytosis) standards, including bone marrow examinations with histopathological, cytological and flow cytometric (CD2, CD25) evaluation, KIT D816V mutation and basal serum tryptase level analyses.

The study was approved by the Ethical Commission of the Medical University of Gdańsk, Poland (No. NKEBN/ 151/2010). A written consent from an informed patient was obtained from the study participants.

\section{Collection of Blood Samples}

Tempus Blood RNA Tubes (Applied Biosystems ${ }^{\circledR}$, USA) were used for RNA sampling. All tubes were frozen and stored in $-80{ }^{\circ} \mathrm{C}$ until RNA isolation (maximal period 6 months). RNA was isolated using the Tempus ${ }^{\mathrm{TM}}$ Spin RNA Isolation Kit (Ambion ${ }^{\circledR}$, USA). 
The quality and concentration of RNA were determined using NanoDrop and 2100 Bioanalyzer (Bioanalyzer, BioRad, USA). Only samples with RNA integrity number $>7.5$ were used for further analysis. All RNA samples were stored in $-80{ }^{\circ} \mathrm{C}$ until Reverse Transcription PCR (maximal period 12 months).

Reverse transcription was performed using the High Capacity cDNA Reverse Transcription Kits RNase Inhibitor (Invitrogen ${ }^{\mathrm{TM}}$, USA), which delivers extremely highquality, single-stranded cDNA $0.02-2 \mu \mathrm{g}$ total RNA. Reactions were scaled up to $100 \mu \mathrm{L}$ to generate $10 \mu \mathrm{g}$ of cDNA from a single reaction. The cDNA samples were stored in $-20{ }^{\circ} \mathrm{C}$ until real-time RT-PCR (period 2-6 months).

\section{Gene Expression}

Studied genes were chosen based on our previous study on the differences in gene expression among mastocytosis patients with an IVA (Niedoszytko et al. 2011).

Quantitative RT-PCRs of 11 genes plus ribosomal 18S RNA were run using TaqMan ${ }^{\circledR}$ Array Micro Fluidic Cards (Applied Biosystems, USA) in 7900HT Fast Real-Time PCR system with preinstalled TaqMan ${ }^{\circledR}$ Array Micro Fluidic Card Thermal Cycling Block, according to the manufacturer's protocol.

One channel of a microfluidic card was loaded with a mix of $45 \mu \mathrm{l}$ RNA-ase free water (Nuclease-Free Water (not DEPC-Treated) Ambion $^{\circledR}$, USA), $50 \mu \mathrm{L}$ TaqMan $^{\circledR}$ Master Mix and $5 \mu$ of cDNA to obtain $25 \mathrm{ng}$ cDNA in the reaction mix. This cDNA template corresponded to $25 \mathrm{ng}$ of total RNA.

\section{Data Analysis}

Relative gene expression values were calculated using the $\Delta \Delta \mathrm{Ct}$ method (Schefe et al. 2006) using the Sequence Detection System 2.2.1 software (Applied Biosystems, USA). The $\Delta \Delta \mathrm{Ct}$ method gave the amount of the target gene normalized to an endogenous reference gene and relative to a calibrator sample (reference for all samples). The raw gene expression values were normalized according to the expression of ribosomal 18S RNA. The normalized expression of each gene was calibrated by its expression in a virtual calibration sample.

A statistical analysis was performed using Statistica v.10 StatSoft (Tulsa, USA). In the statistical analysis, the Mann-Whitney $U$ test, Students $t$ test, Wilcoxon test, Pearson correlation test and logistic regression analysis were used.

$p$ values less than 0.05 were considered to be associated with statistical significance.

\section{Food and Drug Hypersensitivity and IVA Diagnosis and Treatment}

Patients were asked for symptoms of anaphylactic reactions during a standard diagnosis and, additionally, a questionnaire focused on symptoms of insect, drug and food hypersensitivity was used. The skin prick test and the specific immunoglobulin E (sIgE) measurement where performed in cases where the immunological mechanism of an allergy was suspected. The food challenge was not used due to the high risk of an anaphylactic reaction in mastocytosis patients. IVA was diagnosed in accordance with European Academy of Allergy and Clinical Immunology (EAACI) guidelines and included specific IgE evaluation, and both skin and intracutaneous tests in all patients in concordance with the symptoms of IVA in medical history. The drug hypersensitivity (non-steroid anti-inflammatory drugs, antibiotics, local anesthetics) was diagnosed in accordance with EAACI/ENDA (European Network for Drug Allergy) guidelines and was confirmed by both skin prick and/or intracutaneous tests which were followed by the drug provocation tests (DPT) in selected group of patients. Before DPT, the evaluation of the individual risk-benefit ratio was estimated. DPTs were performed with all precaution measures in the inpatient clinic. DPTs were positive if they reproduced the original symptoms or objective symptoms of intolerance as urticaria or a drop of at least $20 \%$ in FEV1 in spirometry.

Patients were treated according to the ECNM standards. In the event of food or drug hypersensitivity, avoidance of the culprit allergen was advised. Venom immunotherapy was initiated in ten patients $(76.9 \%)$ with confirmed IVA $(n=13)$. The other three patients $(23.1 \%)$ are due to start venom immunotherapy in the near future. The treatment was performed according to an ultrarush (wasp) or rush (bee) protocol $\mu$ with the maintenance dose of $100 \mu \mathrm{g}$. According to recommendations, this therapy should be performed lifelong in mastocytosis patients. Patients were trained in self-management of anaphylaxis, avoidance of hidden allergen sources and cross reacting allergens.

\section{Results}

The whole ( $n=57$ ) study group included 23 patients with cutaneous mastocytosis (CM; $40 \%), 32$ with ISM (56\%), 2 patients with smouldering systemic mastocytosis (4\%). The symptoms of food or drug hypersensitivity or IVA were found in 28 subjects (49\%), while 29 patients (51\%) were free of such symptoms (Fig. 1).

Symptoms of food hypersensitivity were found in 12 patients $(21 \%)$, including 3 patients (13\%) with CM, and $9(28 \%)$ with ISM. The factors responsible for the 


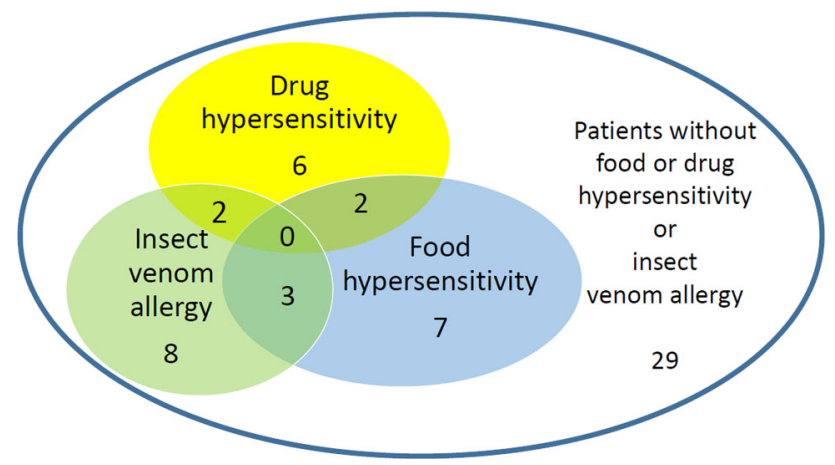

Fig. 1 The prevalence of food and drug hypersensitivity and insect venom allergy in studied mastocytosis patients

symptoms were (number of patients in brackets): fish (4), milk (3), chocolate (3), red wine/alcohol (3), apple (3), nuts (3), orange (2), banana (2), strawberries (2), meat (2). IgEmediated food allergy was diagnosed in 3 ISM patients (5.2\%).

IVA was confirmed in 13 patients (22.8\%) including 6 patients $(10.5 \%)$ with $\mathrm{CM}$ and 7 patients $(12.3 \%)$ with ISM.

Drug hypersensitivity was diagnosed in ten patients $(17.5 \%)$ including six patients $(10.5 \%)$ and four patients (7\%) with ISM. Drugs causing allergic reactions included nonsteroidal anti-inflammatory drugs $(n=6)$, antibiotics $(n=2)$, local anesthetics $(n=3)$.

The prevalence of food and drug hypersensitivity and IVA is presented in Fig. 1. None of the patients presented all types of studied hypersensitivity and allergy. Food and drug hypersensitivity were found in two patients, IVA occurred in two patients with drug and two patients with food hypersensitivity.

No differences in tryptase level, KIT mutation, CD2 and CD 25 expression were observed according to the presence of food and drug intolerance, and IVA.

Significant differences in gene expression were found for TRAF4 $(p=0.008)$ in the comparison of the mastocytosis patients with and without concomitant food hypersensitivity (Table 1 ). The difference was significant in ISM $(p=0.04)$, not CM patients. It was also significant in the sub-group of patients with a food allergy $(p=0.04)$.

Moreover, significant differences in gene expression were found for B3GAT1 $(p=0.003)$ in patients with IVA compared to patients who did not react to an insect sting. B3GAT1 gene was underexpressed in patients with anaphylaxis (Table 2). There were no statistical significant differences in gene expression profiles depending on the history of drug hypersensitivity in the medical history.
Table 1 Gene expression in patients with mastocytosis with food hypersensitivity compared to patients without symptoms of food hypersensitivity

\begin{tabular}{ll}
\hline Gen & $p$ value \\
\hline ABI3BP-Hs00227206_m1 & 0.91 \\
ANKRD6-Hs00208902_m1 & 0.03 \\
B3GAT1-Hs00218629_m1 & 0.09 \\
CDC42BPA-Hs00177522_m1 & 0.24 \\
DVL1-Hs00182896_m1 & 0.08 \\
G0S2-Hs00274783_s1 & 0.17 \\
HLA-DRB4-Hs03027795_uH & 0.44 \\
JUP-Hs00158408_m1 & 0.08 \\
MAP2K3-Hs00177127_m1 & 0.13 \\
TNFRSF4-Hs00533968_m1 & 0.09 \\
TRAF4-Hs00188755_m1 & $0.008^{*}$
\end{tabular}

* Statistically significant difference

Table 2 Gene expression in patients with IVA compared to patients without IVA

\begin{tabular}{ll}
\hline Gen & $p$ value \\
\hline ABI3BP-Hs00227206_m1 & 0.87 \\
ANKRD6-Hs00208902_m1 & 0.64 \\
B3GAT1-Hs00218629_m1 & $0.003^{*}$ \\
CDC42BPA-Hs00177522_m1 & 0.86 \\
DVL1-Hs00182896_m1 & 0.51 \\
G0S2-Hs00274783_s1 & 0.51 \\
HLA-DRB4-Hs03027795_uH & 0.62 \\
JUP-Hs00158408_m1 & 0.47 \\
MAP2K3-Hs00177127_m1 & 0.07 \\
TNFRSF4-Hs00533968_m1 & 0.24 \\
TRAF4-Hs00188755_m1 & 0.50 \\
\hline
\end{tabular}

* Statistically significant difference

\section{Discussion}

The results of the study indicate the increased expression of TRAF4 in mastocytosis patients with food hypersensitivity and decreased expression of B3GAT1 in mastocytosis patients with IVA. There were no significant differences in gene expression in patients with mastocytosis depending on the occurrence of drug hypersensitivity. The symptoms of IVA were found in $12(21 \%)$ of subjects; food hypersensivity in $12(21 \%)$ of patients and drug hypersensitivity in $10(17 \%)$ of mastocytosis patients. Interestingly, the cooccurrence of studied food and drug hypersensitivity or IVA was found only in seven cases. It may be the reason for the differences in gene expression indicating the role of 
TRAF4 in food hypersensitivity and B3GAT1 in IVA as the patient groups and the pathophysiology of the reaction is different.

TRAF4 is crucial in developmental and morphogenic processes involved in oncogenesis and inflammation (Rousseau et al. 2011). Except the high differences in the expression of TRAF4 between control and neoplastic cells, the differences in inflammatory diseases like Crohn disease (Marinis et al. 2011; Rousseau et al. 2011) or airway inflammation (Zepp et al. 2015) were described. Recent studies indicate that TRAF4-mediated SMURF2-dependent degradation of DAZAP2 is a crucial step in interleukin (IL)-25 signalling. IL-25 is the only member of IL-17 cytokine family involved in Th2-associated cytokine production (IL-4, IL-5, IL-9 and IL-13), eosinophil recruitment, $\operatorname{IgE}$ production and innate immune response (Zepp et al. 2015). These mechanisms are common in allergic diseases including food allergy, thus, a higher TRAF4 expression may increase the risk of food hypersensitivity. Our data support the identification of the increased TRAF4 expression in airway inflammation (Zepp et al. 2015), Crohn disease (Marinis et al. 2011; Rousseau et al. 2011) and indicate the need for further studies.

B3GAT1 gene is localized on 11q25 chromosome and is a member of the glucuronyltransferase gene family. This gene product functions as the key enzyme in a glucuronyl transfer reaction during the biosynthesis of the carbohydrate epitope HNK-1, known also as CD57. It acts to add a glucuronic acid to the terminal $N$-acetyllactosamine (Lac) disaccharide to form the HNK-1 epitope precursor. CD57positive $\mathrm{T}$ cells are important effectors of immune regulation and cytotoxicity. Olloquequi et al. (2011) found a significant and specific increase in the follicular density of $\mathrm{CD}^{+} 7^{+}$cells in patients with chronic obstructive pulmonary disease (COPD), which support the hypothesis of local immune dysfunction. Furthermore, this study proved that COPD patients exhibit a significant increase in the follicular density of $\mathrm{CD}^{+} 7^{+}$cells compared to nonsmokers and smokers without COPD. This finding shed light on the role that lymphoid folliculles could play in the disease, since there is an evidence pointing toward CD57 as a marker of lung inflammation (Palmer et al. 2005) or even as a marker of general immune dysfunction independent of the underlying disease (Focosi et al. 2010). Moreover, it has recently been demonstrated that CD57 antigen is also a marker of terminally differentiated cells with a high cytolytic potential (Chattopadhyay et al. 2009). It is considered that CD57 expression on $\mathrm{CD}^{+}{ }^{+} \mathrm{T}$ cells, $\mathrm{CD} 4^{+} \mathrm{T}$ cells, and NK cells is a general marker of proliferative inability, a history of more cell divisions, and short telomeres (Brenchley et al. 2003). CD57 is involved in apoptosis and lack of proliferation. Moreover, the proliferative defects and apoptotic nature observed within HIV-specific $\mathrm{CD}^{+} \mathrm{T}$ cells can be predicted by expression of CD57 on cells. The presence of these cells, however, does not reflect a defect particular to the immune response to HIV or an effect limited to any particular virus, but simply reflects the normal consequence of persistent immune activation (Brenchley et al. 2003).

Our previous data indicated that genes involved in pathways responsible for the development of cancer may be related to the risk of an anaphylactic reaction to insect venom. We hypothesized that a more pronounced mast cells dysfunction in patients without a history of anaphylaxis is present (Niedoszytko et al. 2011). The current study is performed in a group of patients with less advanced mastocytosis, also including CM patients. Thus, the differences in gene expression responsible for a neoplastic transformation were not found. The observation of a higher expression of TRAF4 related to Th2 inflammation confirms another observation of the role of the IL-13 gene polymorphism in the pathogenesis of mastocytosis and frequent symptoms of food hypersensitivity affecting this group of patients (Nedoszytko et al. 2009). The genetics of anaphylaxis and intolerance are poorly understood. New observations describing the role of calcitonin gene-related peptide, the neurotransmitter of enteric sensory neurons responsible for the microtubule organization and interaction between mucosal mast cells and the development of food allergy, prove that food hypersensitivity mechanisms involve more pathways than the classic IgE response (Kim et al. 2014). The identification of novel targets like TRAF4 may constitute a research field focused on new therapeutic targets (Kim et al. 2014).

The opinions concerning food hypersensitivity in mastocytosis were conflicting. Studies on skin prick test and sIgE levels in mastocytosis patients do not show higher prevalence than in the general population (González de Olano et al. 2007). Similarly, the proportion of patients with an elevated tryptase level is higher in patients with an IVA than in subjects suffering from a food allergy (Bonadonna et al. 2009a, b). The studies based on the symptoms and broader mechanisms of food hypersensitivity, including histamine hypersensitivity (Brockow et al. 2008; Cifuentes et al. 2013; Koga et al. 2011; Prieto-García et al. 2015; Vlieg-Boerstra et al. 2005), present clinically important systemic reactions in this group of patients. Our results confirm that the prevalence of food hypersensitivity in mastocytosis patients $(21 \%)$ is higher than in the comparable age group of adults in Poland (5\%) (Samolinski 2015). The present study also adds a genetic trace in the pathology of the symptoms described as important by half of the patients (Jennings et al. 2014). The mechanism of hypersensitivity may be related to the TRAF4-mediated SMURF2-dependent degradation of DAZAP2 (Zepp et al. 2015), irrespective of the type of 
hypersensitivity (allergic or non-allergic). The variety of symptoms caused by food hypersensitivity and the treatment methods, including food avoidance, indicates the crucial role of a dietitian and patient education. In contrast, the IVA is mediated by IgE-dependent allergy thus the lifelong insect venom immunotherapy is crucial to prevent fatal reactions.

In conclusion, the TRAF4 expression was higher in mastocytosis patients with food hypersensitivity in their medical history. B3GAT1 gene and antigen CD57 play an important role in immune system and neoplasm processes. CD57 is a marker of well-differentiated cells with high cytotoxic potential. Moreover, CD57 is a marker of the immune system dysfunction regardless of the underlying disease. CD57 lymphocytes are involved in many chronic processes associated with the activation of immune system, such as viral infections, inflammatory disorders and also neoplasms, as well as physical stress and aging. The variation in the glycosylation of plasma proteins caused by the polymorphisms of B3GAT1 could be a predisposing or prognostic factor in numerous diseases.

Acknowledgments The study was supported by Grant No. 2011/01/ M/NZ501362 of the Polish National Science Centre.

\section{Compliance with ethical standards}

Conflict of interest The authors do not declare any conflicts of interest.

Open Access This article is distributed under the terms of the Creative Commons Attribution 4.0 International License (http:// creativecommons.org/licenses/by/4.0/), which permits unrestricted use, distribution, and reproduction in any medium, provided you give appropriate credit to the original author(s) and the source, provide a link to the Creative Commons license, and indicate if changes were made.

\section{References}

Bonadonna P, Perbellini O, Passalacqua G et al (2009a) Clonal mast cell disorders in patients with systemic reactions to Hymenoptera stings and increased serum tryptase levels. J Allergy Clin Immunol 3:680-686

Bonadonna P, Zanotti R, Pagani M et al (2009b) How much specific is the association between hymenoptera venom allergy and mastocytosis? Allergy 64:1379-1382

Brenchley JM, Karandikar NJ, Betts MR et al (2003) Expression of CD57 defines replicative senescence and antigen-induced apoptotic death of CD8 $+\mathrm{T}$ cells. Blood 101:2711-2720

Brockow K, Jofer C, Behrendt $\mathrm{H}$ et al (2008) Anaphylaxis in patients with mastocytosis: a study on history, clinical features and risk factors in 120 patients. Allergy 63:226-232

Chattopadhyay PK, Betts MR, Price DA et al (2009) The cytolytic enzymes granyzme A, granzyme $\mathrm{B}$, and perforin: expression patterns, cell distribution, and their relationship to cell maturity and bright CD57 expression. J Leukoc Biol 85:88-97
Cifuentes L, Ring J, Brockow K (2013) Clonal mast cell activation syndrome with anaphylaxis to sulfites. Int Arch Allergy Immunol 162:94-96

Focosi D, Bestagno M, Burrone O et al (2010) CD57+ T lymphocytes and functional immune deficiency. J Leukoc Biol 87:107-116

González de Olano D, de la Hoz Caballer B, Núñez López R et al (2007) Prevalence of allergy and anaphylactic symptoms in 210 adult and pediatric patients with mastocytosis in Spain: a study of the Spanish network on mastocytosis (REMA). Clin Exp Allergy 37:1547-1555

Górska A, Niedoszytko M, Lange M et al (2015) Risk factors for anaphylaxis in patients with mastocytosis. Pol Arch Med Wewn 125:46-53

Henderson WA, Shankar R, Taylor TJ et al (2012) Inverse relationship of interleukin-6 and mast cells in children with inflammatory and non-inflammatory abdominal pain phenotypes. World J Gastrointest Pathophysiol 3:102-108

Jennings S, Russell N, Jennings B et al (2014) The Mastocytosis Society survey on mast cell disorders: patient experiences and perceptions. J Allergy Clin Immunol Pract 2:70-76

Kim JH, Yamamoto T, Lee J et al (2014) CGRP, a neurotransmitter of enteric sensory neurons, contributes to the development of food allergy due to the augmentation of microtubule reorganization in mucosal mast cells. Biomed Res 35:285-293

Koga H, Kokubo T, Akaishi M et al (2011) Neonatal onset diffuse cutaneous mastocytosis: a case report and review of the literature. Pediatr Dermatol 28:542-546

Ludolph-Hauser D, Ruëff F, Fries C et al (2001) Constitutively raised serum concentrations of mast-cell tryptase and severe anaphylactic reactions to Hymenoptera stings. Lancet 357:361-362

Marinis JM, Homer CR, McDonald C, Abbott DW et al (2011) A novel motif in the Crohn's disease susceptibility protein, NOD2, allows TRAF4 to down-regulate innate immune responses. J Biol Chem 286:1938-1950

Nedoszytko B, Niedoszytko M, Lange M et al (2009) Interleukin-13 promoter gene polymorphism $-1112 \mathrm{C} / \mathrm{T}$ is associated with the systemic form of mastocytosis. Allergy 64:287-294

Niedoszytko M, Bruinenberg M, van Doormaal JJ et al (2011) Gene expression analysis predicts insect venom anaphylaxis in indolent systemic mastocytosis. Allergy 66:648-657

Nwaru BI, Hickstein L, Panesar SS et al (2014) The epidemiology of food allergy in Europe: a systematic review and meta-analysis. Allergy 69:62-75

Olloquequi J, Montes JF, Prats A et al (2011) Significant increase of CD57 cells in pulmonary lymphoid follicles of COPD patients. Eur Respir J 37:289-298

Palmer BE, Blyveis N, Fontenot AP et al (2005) Functional and phenotypic characterization of CD57+ CD4+ T cells and their association with HIV-1-induced T cell dysfunction. J Immunol 175:8415-8423

Peavy R, Metcalfe D (2008) Understanding the mechanisms of anaphylaxis. Curr Opin Allergy Clin Immunol 8:310-315

Prieto-García A, Álvarez-Perea A, Matito A et al (2015) Systemic mastocytosis presenting as IgE-mediated food-induced anaphylaxis: a report of two cases. J Allergy Clin Immunol Pract 3:456-458

Ring J (2014) What is an allergy? In: Akids C, Agache I (eds) Global atlas of allergy. European Academy of Allergy and Clinical Immunology, EAACI, Zurich

Rousseau A, Rio MC, Alpy F (2011) TRAF4, at the crossroad between morphogenesis and cancer. Cancers 3:2734-2749

Samolinski B (2015) Epidemiology of Allergic Diseases in Poland. Available via ECAP. http://ecap.pl/pdf/ECAP_wyniki_pl.pdf. Accessed 1 July 2015 
Schefe JH, Lehmann KE, Buschmann IR et al (2006) Quantitative real-time RT-PCR data analysis: current concepts and the novel "gene expression's CT difference" formula. J Mol Med 84:901-910

Valent P, Akin C, Escribano L et al (2007) Standards and standardization in mastocytosis: consensus statements on diagnostics, treatment recommendations and response criteria. Eur J Clin Invest 37:435-453

Valent P, Akin C, Arock M et al (2012) Definitions, criteria and global classification of mast cell disorders with special reference to mast cell activation syndromes: a consensus proposal. Int Arch Allergy Immunol 157:215-225 van Doormaal JJ, Arends S, Brunekreeft KL et al (2013) Prevalence of indolent systemic mastocytosis in a Dutch region. J Allergy Clin Immunol 131:1429-1431

Vlieg-Boerstra BJ, van der Heide S, Oude Elberink JN et al (2005) Mastocytosis and adverse reactions to biogenic amines and histamine-releasing foods: what is the evidence? Neth J Med 63:244-249

Zepp JA, Wu L, Qian W et al (2015) TRAF4-SMURF2-mediated DAZAP2 degradation is critical for IL-25 signaling and allergic airway inflammation. J Immunol 194:2826-2837 